\title{
Residential Care Community Resident Characteristics: United States, 2018
}

\author{
Christine Caffrey, Ph.D., Manisha Sengupta, Ph.D., and Amanuel Melekin, Ph.D.
}

\section{Key findings}

\section{Data from the National Study of Long-Term Care Providers}

- In 2018, most residential care community residents were female (67\%), non-Hispanic white $(89 \%)$, and aged 85 and over $(55 \%)$.

- The percentage of residents with Medicaid varied by age. Overall, $19 \%$ of residential care community residents were Medicaid beneficiaries.

- Residential care community residents needed the most assistance with bathing, walking, and dressing; $61 \%$ of residents needed assistance with three or more activities of daily living.

- Among residential care community residents, $55 \%$ were diagnosed with high blood pressure, and 34\% were diagnosed with Alzheimer disease and other dementias.
Residential care communities provide housing for persons who cannot live independently but generally do not require the skilled care provided by nursing homes. On any given day in 2018, an estimated 918,700 residents lived in residential care communities $(1,2)$. With the aging of the U.S. population, the numbers of residential care community residents will likely increase, becoming a substantial segment of the long-term care population. This report presents national estimates of selected characteristics of residential care community residents in 2018 .

\section{In 2018, most residential care community residents were female, non-Hispanic white, and aged 85 and over.}

- Residential care community residents were $67 \%$ female and $33 \%$ male (Figure 1).

Figure 1. Sex, race and ethnicity, and age of residential care community residents: United States, 2018

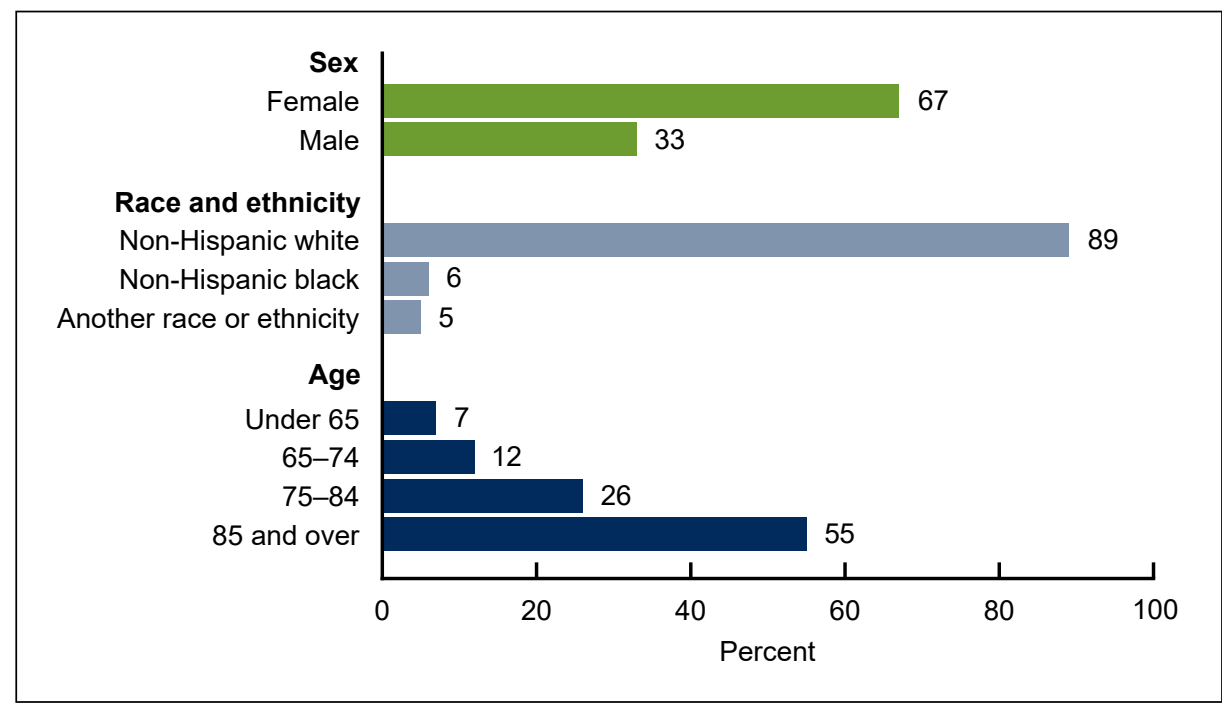

NOTES: Another race or ethnicity includes Hispanic, Latino, or Spanish origin or descent; non-Hispanic American Indian or Alaska Native; non-Hispanic Asian; non-Hispanic Native Hawaiian or Other Pacific Islander; non-Hispanic more than one race; and non-Hispanic unknown. Figure excludes cases with missing data. See Data source and methods in this report for details. Access non-Hispanic unknown. Figure excludes cases with missing data. See Data source and
data table for Figure 1 at: https://www.cdc.gov/nchs/data/databriefs/db404-tables.pdf\#1. SOURCE: National Center for Health Statistics, National Study of Long-Term Care Providers, 2018. 
- Almost 9 in 10 residential care community residents were non-Hispanic white (89\%), 6\% were non-Hispanic black, and 5\% were of another race or ethnicity.

- Most residential care community residents were aged 85 and over (55\%), 38\% were aged $65-84$, and $7 \%$ were under age 65 .

\section{Almost 2 in 10 residential care community residents were Medicaid beneficiaries, and the percentage of residents with Medicaid varied by age.}

- Overall, $19 \%$ of residential care community residents were Medicaid beneficiaries (Figure 2).

- By age, $11 \%$ of residents aged 85 and over, $21 \%$ of residents aged $75-84,38 \%$ of residents aged $65-74$, and $50 \%$ of residents under age 65 were Medicaid beneficiaries.

Figure 2. Residential care community residents with Medicaid, by age: United States, 2018

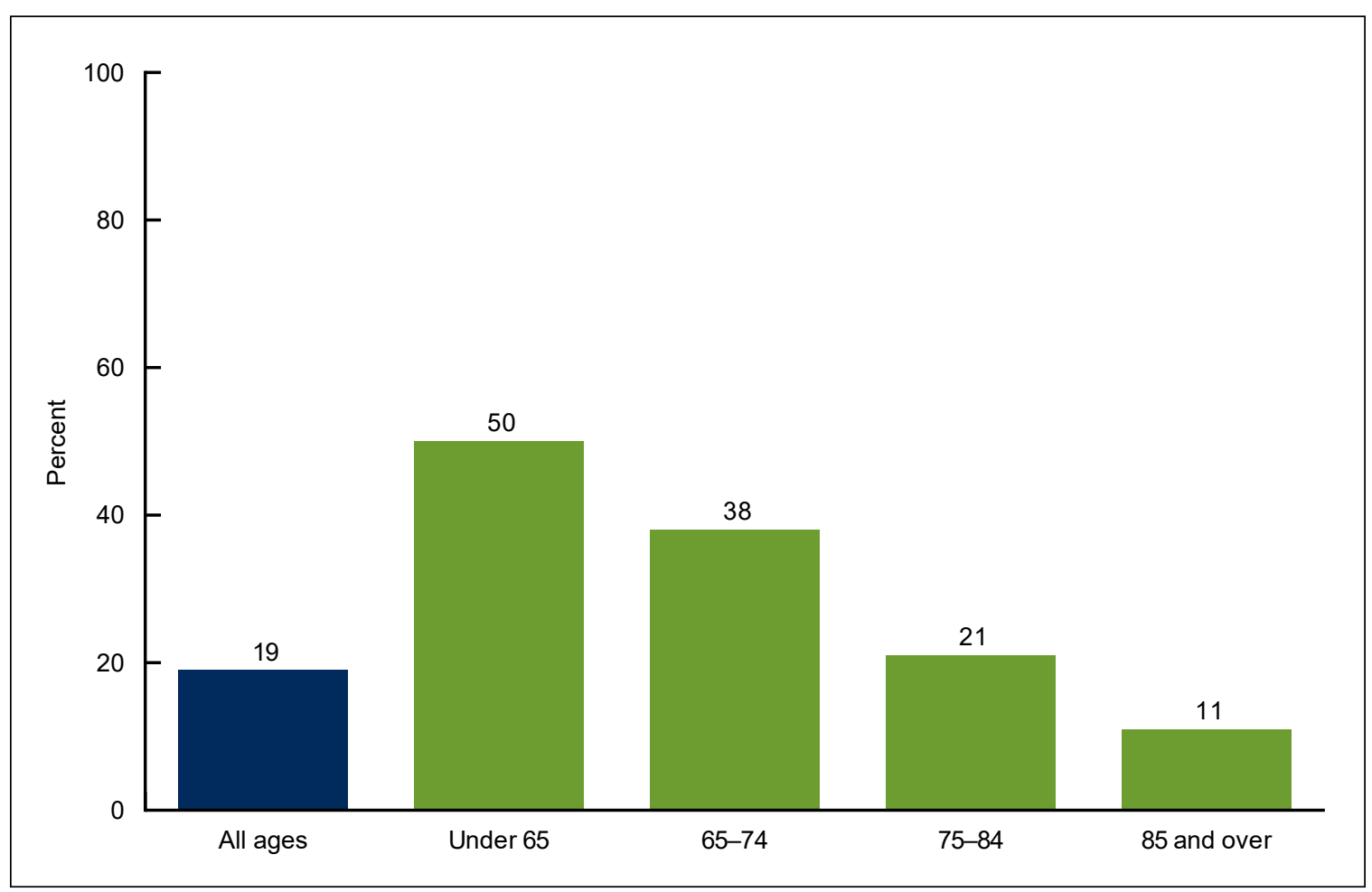

NOTES: Medicaid includes residents who had some or all of their long-term care services paid by Medicaid during the last complete month prior to the survey. Figure excludes cases with missing data. See Data source and methods in this report for details. Access data table for Figure 2 at: https://www.cdc.gov/nchs/data/ databriefs/db404-tables.pdf\#2.

SOURCE: National Center for Health Statistics, National Study of Long-Term Care Providers, 2018. 


\section{NCHS Data Brief a No. 404 a September 2021}

\section{Residential care community residents needed the most assistance with bathing, walking, and dressing; almost two-thirds of residents needed assistance with three or more activities of daily living (ADLs).}

- Among residential care community residents, $61 \%$ needed assistance with three or more ADLs, 27\% needed assistance with one or two ADLs, and 12\% did not need assistance with any ADLs (Figure 3).

- Residential care community residents needed assistance with bathing (77\%), walking $(69 \%)$, dressing (62\%), transferring in and out of a bed or chair $(51 \%)$, toileting $(49 \%)$, and eating $(26 \%)$.

Figure 3. Residential care community residents needing assistance with activities of daily living: United States, 2018

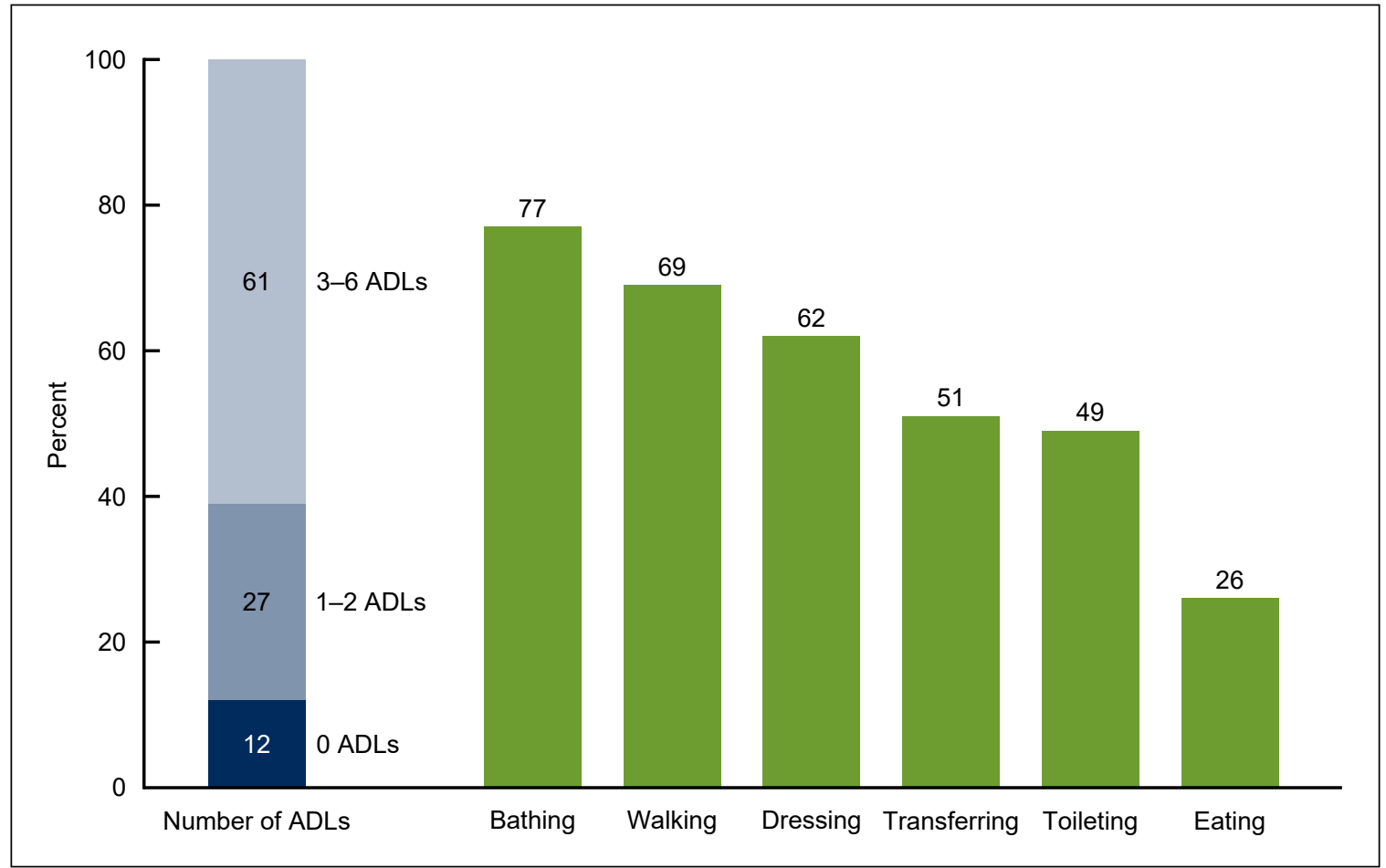

NOTES: Activities of daily living (ADLs) refers to residents needing any help or supervision from another person, use of assistive devices, or both. Walking includes using a cane, walker, or wheelchair, or help from another person. Transferring includes needing assistance getting in and out of a bed or chair. Individual ADLs are not mutually exclusive; a resident can be counted for more than one ADL. Figure excludes cases with missing data. See Data source and methods in this report for details. Access data table for Figure 3 at: https://www.cdc.gov/nchs/data/databriefs/db404-tables.pdf\#3.

SOURCE: National Center for Health Statistics, National Study of Long-Term Care Providers, 2018. 
NCHS Data Brief a No. 404 a September 2021

\section{About one-half of residential care community residents were diagnosed with high blood pressure, and about one-third were diagnosed with Alzheimer disease and other dementias.}

- Seventeen percent of residential care community residents had ever been diagnosed with 4-10 of the most common chronic conditions, $49 \%$ had ever been diagnosed with $2-3$ of the most common chronic conditions, $20 \%$ had ever been diagnosed with one of these chronic conditions, and the remaining $14 \%$ had never been diagnosed with these conditions (Figure 4).

- The 10 most common chronic conditions among residential care community residents were high blood pressure (55\%), Alzheimer disease and other dementias (34\%), depression $(27 \%)$, arthritis $(20 \%)$, diabetes $(20 \%)$, heart disease $(17 \%)$, osteoporosis $(12 \%)$, chronic obstructive pulmonary disease and allied conditions (11\%), stroke (10\%), and cancer (9\%).

Figure 4. Most common chronic conditions among residential care community residents: United States, 2018

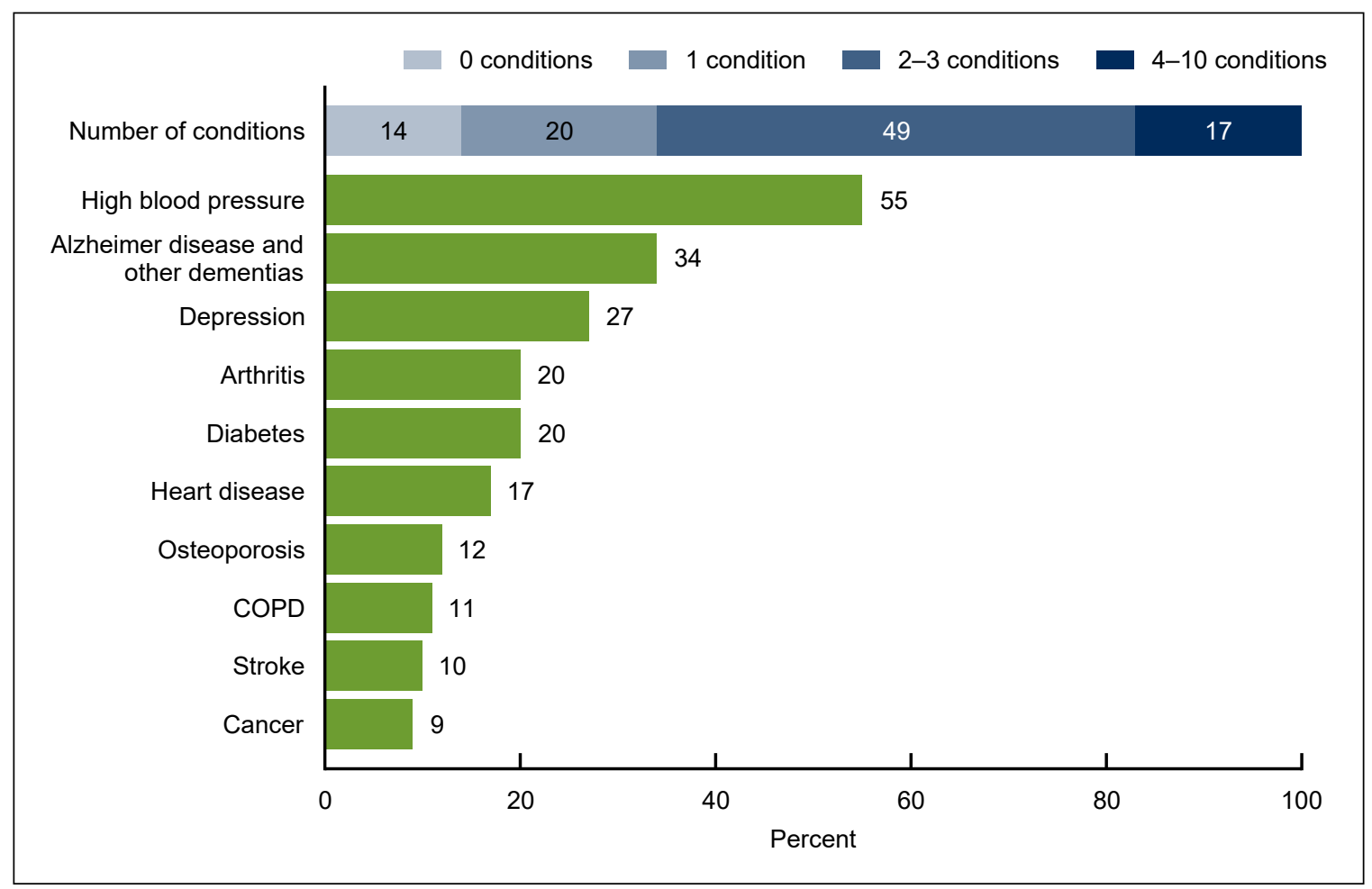

NOTES: Heart disease includes coronary or ischemic heart disease. COPD stands for chronic obstructive pulmonary disease and includes chronic bronchitis or emphysema. Individual conditions are not mutually exclusive; a resident can be counted for more than one condition. Figure excludes cases with missing data. See Data source and methods in this report for details. Access data table for Figure 4 at: https://www.cdc.gov/nchs/data/databriefs/db404-tables.pdf\#4. SOURCE: National Center for Health Statistics, National Study of Long-Term Care Providers, 2018. 


\section{Summary}

In 2018 in the United States, the resident population living in residential care communities were mostly female (67\%), non-Hispanic white (89\%), and aged 85 and over (55\%). Overall, 19\% of residential care community residents were Medicaid beneficiaries, and residents under age 65 made up the largest percentage of Medicaid beneficiaries (50\%). Residential care community residents needed the most assistance with bathing (77\%), walking (69\%), and dressing (62\%), and a substantial percentage were diagnosed with Alzheimer disease and other dementias (34\%) and depression (27\%).

This report presents the most current national estimates of selected characteristics of residents in residential care communities. This brief profile of residential care community residents provides information to policy makers, providers, researchers, and consumer advocates as they plan how to best meet the needs of an aging population.

\section{Definitions}

Assistance with selected activities of daily living (ADLs): Refers to residents needing any help or supervision from another person, use of assistive devices, or both, with limitations in up to six ADLs (bathing; walking - includes using a cane, walker, or wheelchair, or help from another person; dressing; transferring in and out of a bed or chair; toileting; and eating) that reflect a resident's capacity for self-care.

Chronic obstructive pulmonary disease (COPD): Includes chronic obstructive pulmonary disease and chronic bronchitis or emphysema.

Heart disease: Includes coronary or ischemic heart disease.

Medicaid beneficiaries: Refers to residents who had some or all of their long-term care services paid by Medicaid during the last complete month prior to the survey.

Race or ethnicity: Based on responses to two questions that determine Hispanic or Latino origin and race. Three categories were constructed: non-Hispanic white, non-Hispanic black, and another race or ethnicity. Another race or ethnicity includes persons of Hispanic, Latino, or Spanish origin or descent; non-Hispanic American Indian or Alaska Native persons; non-Hispanic Asian persons; non-Hispanic Native Hawaiian or Other Pacific Islander persons; non-Hispanic persons of more than one race; and non-Hispanic persons of unknown race.

Residential care communities: Includes assisted living communities and other residential care communities (for example, personal care homes, adult care homes, board care homes, or adult foster care) that meet the study eligibility criteria. More details about the study eligibility criteria are published elsewhere $(1,2)$. 


\section{NCHS Data Brief a No. 404 - September 2021}

\section{Data source and methods}

Data for this report are from the residential care community survey component of the 2018 wave of the biennial National Study of Long-Term Care Providers (NSLTCP), conducted by the National Center for Health Statistics (NCHS). The survey used a sample of residential care community residents, obtained from a frame that was constructed from lists of licensed residential care communities acquired from the state licensing agencies in each of the 50 states and the District of Columbia. More details about NSLTCP, including eligibility criteria, design, and outcomes, are published elsewhere $(1,2)$.

The percentage of cases with missing data for variables in this report ranged from $0.2 \%$ for Medicaid to $3.2 \%$ for needing assistance with walking. These cases were excluded from the analyses on a variable-by-variable basis. Data analyses were performed using the following statistical packages: SAS version 9.3 (3), SAS-callable SUDAAN version 11.0.0 (4), and STATA/ $\mathrm{SE}$ version 12.1 (5). Analyses incorporated complex survey weights. 


\section{About the authors}

Christine Caffrey, Manisha Sengupta, and Amanuel Melekin are with NCHS' Division of Health Care Statistics, Long-Term Care Statistics Branch.

\section{References}

1. National Center for Health Statistics. 2018 National Study of Long-Term Care Providers survey methodology for the Adult Day Services Center and Residential Care Community components. 2021. Available from: https://www.cdc.gov/nchs/npals/questionnaires.htm.

2. National Center for Health Statistics. 2018 National Study of Long-Term Care Providers (NSLTCP) Residential Care Communities Survey public-use data file: Data description and usage (readme). 2021. Available from: https://www.cdc.gov/nchs/npals/questionnaires.htm.

3. SAS Institute. SAS statistical software (Release 9.3) [computer software]. 2011.

4. RTI International. SAS-callable SUDAAN statistical software (Release 11) [computer software]. 2012.

5. StataCorp. Stata statistical software (Release 14) [computer software]. 2015. 
Centers for Disease Control and Prevention

\section{NCHS Data Brief a No. 404 a September 2021}

Keywords: assisted living •long-term services and supports $\bullet$ National Study of Long-Term Care Providers

\section{Suggested citation}

Caffrey C, Sengupta M, Melekin A. Residential care community resident characteristics: United States, 2018. NCHS Data Brief, no 404. Hyattsville, MD:

National Center for Health Statistics. 2021. DOI: https://doi.org/10.15620/cdc:103826.

\section{Copyright information}

All material appearing in this report is in the public domain and may be reproduced or copied without permission; citation as to source, however, is appreciated.

\section{National Center for Health Statistics}

Brian C. Moyer, Ph.D., Director Amy M. Branum, Ph.D., Associate Director for Science

\section{Division of Health Care Statistics} Carol J. DeFrances, Ph.D., Acting Director Alexander Strashny, Ph.D., Associate Director for Science

For e-mail updates on NCHS publication releases, subscribe online at: https://www.cdc.gov/nchs/email-updates.htm.

For questions or general information about NCHS:

Tel: 1-800-CDC-INFO (1-800-232-4636)

TTY: $1-888-232-6348$

Internet: https://www.cdc.gov/nchs

Online request form: https://www.cdc.gov/info

ISSN 1941-4927 Print ed.

ISSN 1941-4935 Online ed. 